\title{
25 Research Square \\ Exploring the bacterial community composition of soil from a tropical dry evergreen forest in Tamil Nadu, India
}

\section{Saptashwa Datta}

SRMIST: SRM Institute of Science and Technology

Melvin S. Samuel

Indian Institute of Technology Kharagpur

Ethiraj Selvarajan ( $\nabla$ selrajan@gmail.com )

SRMIST: SRM Institute of Science and Technology https://orcid.org/0000-0002-3447-6432

\section{Research Article}

Keywords: Metagenomics, omics, bacterial communities, tropical evergreen forests

Posted Date: April 15th, 2021

DOI: https://doi.org/10.21203/rs.3.rs-218538/v1

License: (c) (i) This work is licensed under a Creative Commons Attribution 4.0 International License.

Read Full License 


\section{Abstract}

Metagenomics is a cutting edge omics technology that has been employed in various fields including novel product discovery, diagnostics, and pollutant monitoring. 16S metagenome amplicon sequencing is used for understanding the microbial diversity from various environments. Forest ecosystems have been known for the discovery of novel bacteria and also bacteria that produces novel compounds that are pharmaceutically and industrially relevant. In this study we try to show the bacterial community structure of the soil obtained from a tropical evergreen forest in India. We use 16s metagenomics sequencing and then follow it up with various analysis like alpha diversity analysis, to find out the dominant bacterial species found in these soils. Actinobacteria was found to be the most copmmonly found bacterial phylum followed by proteobacteria, firmicutes, chloroflexi, acidobacteria, verrucomicrobia, bacteroidetes, gemmatimonadetes, nitospirae and other unclassified organisms. Further studies can elucidate on the discovery of novel compounds from these bacteria.

\section{Introduction}

Omics based technologies have become very important for understanding life, its various functions and the marked differences. Metagenomics is one of the recent upcoming fields of omics technologies that have been utilized in a wide number of fields including the discovery of novel enzymes, diagnosis of pathogenic microbes, environmental pollutant monitoring, and discovery of novel therapeutic compounds. This omics technology involves the extraction of the total DNA from various samples and then sequencing it[1]. To understand the species diversity in various environments a $16 \mathrm{~s}$ metagenomic sequencing is performed[2]. The tropical dry evergreen forests are home to a lot of endemic plant and animal species. The forests are known to have a wide diversity of life and much of it still remains unexplored. The various species of plants that are found in these forests also include various important medicinal plants. A lot of the forest land has been cleared to give way to human habitation. The rest of our remaining forest land should be well conserved and hence it is very important to improve our understanding of these forests[3]. The dry evergreen forests are at an altitude that measures about 2001500 metres and receives about $2500-5000 \mathrm{~mm}$ of rainfall. The nutrient composition of the soil of the tropical dry evergreen forests have been studied by research groups. It is suggested that the soil should be high in nutrients as there is continuous supply of fallen leaves which decompose into the soil. The soil was found to contain $273 \mathrm{kgha}^{-1}$ of nitrogen, $21 \mathrm{kgha}^{-1}$ of phosphorus and $143 \mathrm{kgha}^{-1}$ of potassium which are quite high[4]. One of the things that have not been explored is the microbial diversity of these soils. The microbial diversity can help us better understand the soil composition and can also be important to understand the interaction of the bacteria and the plants. In this study we analyze the diversity of bacteria present in the soil taken from tropical evergreen forests in Tamil Nadu by $16 \mathrm{~s}$ metagenomic sequencing.

\section{Materials And Methods}

1.Sample collection 
Soil samples were collected from tropical dry evergreen forest(Vallam Reserve forest) in Chengalpattu, the state of Tamil Nadu in India

\section{Sequencing preparation}

Total genome DNA from samples was extracted using CTAB/SDS method. DNA concentration and purity was monitored on $1 \%$ agarose gels. According to the concentration, DNA was diluted to $1 \mathrm{ng} / \mu \mathrm{L}$ using sterile water. 16S rRNA genes of distinct regions (16SV3-V4) were amplified used specific primer with the barcode. All PCR reactions were carried out with Phusion ${ }^{\circledR}$ High-Fidelity PCR Master Mix (New England Biolabs). Mix same volume of $1 \mathrm{X}$ loading buffer (contained SYBR green) with PCR products and operate electrophoresis on $2 \%$ agarose gel for detection. Samples with bright main strip between $400-450 \mathrm{bp}$ were chosen for further experiments. PCR products was mixed in equidensity ratios. Then, mixture PCR products was purified with Qiagen Gel Extraction Kit (Qiagen, Germany). The libraries generated with NEBNext ${ }^{\circledR}$ UltraTM DNA Library Prep Kit for Illumina and quantified via Qubit and Q-PCR, would be analysed by Illumina platform.

\section{Sequencing data processing and analysis}

Paired-end reads were assigned to samples based on their unique barcode and truncated by cutting off the barcode and primer sequence. Paired-end reads were merged using FLASH (V1.2.7, http://ccb.jhu.edu/software/FLASH/) [5], a very fast and accurate analysis tool, which was designed to merge paired-end reads when at least some of the reads overlap the read generated from the opposite end of the same DNA fragment, and the splicing sequences were called raw tags. Quality filtering on the raw tags were performed under specific filtering conditions to obtain the high-quality clean tags[6] according to the Qiime (V1.7.0, http://qiime.org/scripts/split_libraries_fastq.html)[7] quality controlled process. The tags were compared with the reference database (Gold database, http://drive5.com/uchime/uchime_download.html) using UCHIME algorithm (UCHIME Algorithm, http://www.drive5.com/usearch/manual/uchime_algo.html)[8] to detect chimera sequences (http://www.drive5.com/usearch/manual/chimera_formation.html). And then, the chimera sequences were[9] removed. Then the Effective Tags finally obtained. Sequences analysis was performed by Uparse software (Uparse v7.0.1001 http://drive5.com/uparse/)[10] using all the effective tags. Sequences with $\geq 97 \%$ similarity were assigned to the same OTUs. Representative sequence for each OTU was screened for further annotation. For each representative sequence, Mothur software was performed against the SSUrRNA database of SILVA Database (http://www.arb-silva.de/)[11] for species annotation at each taxonomic rank (Threshold:0.8 1)[12] (kingdom, phylum, class, order, family, genus, species). To get the phylogenetic relationship of all OTUs representative sequences, MUSCLE[13] (Version 3.8.31, http://www.drive5.com/muscle/) was utilised to compare multiple sequences rapidly. OTUs abundance information were normalized using a standard of sequence number corresponding to the sample with the least sequences. Subsequent analysis of alpha diversity was performed basing on this output normalized data. Alpha diversity is applied in analyzing complexity of species diversity for a sample through 6 indices, including Observed-species, Chao1, Shannon, Simpson, ACE, Good's-coverage. All these indices 
for the samples were calculated with QIIME (Version 1.7.0) and displayed with R software (Version 2.15.3).

\section{Results And Discussion}

Amplicon was sequenced using Illumina paired-end chemistry and Illumina platform to generate 250bp paired- end raw reads (Raw PE), and then assembled and pretreated to obtain Clean Tags. The chimeric sequences in Clean Tags were detected and removed to obtain the Effective Tags finally. In order to analyze the species diversity in each sample, all Effective Tags were grouped by $97 \%$ DNA sequence similarity into OTUs (Operational Taxonomic Units).The heat-map shows a view of species composition and abundance among different samples. Figure 1a shows the OTU table heatmap for the sample SN1. Figure 1b shows the SN1 OTU annotation tree construct using GraPhIAn[14]. Specific species (showing the top 10 genus in high relative abundance by default) were selected to make the taxonomy tree Taxonomy tree in sample SN1 is shown in Fig. 2a. KRONA[15] visually displays the analysis result of species annotation. Circles from inside to outside stand for different taxonomic ranks, and the area of sector means respective proportion of different OTU annotation results. The Krona graph for SN1 is shown in Fig. 2b. It is observed that the soil of the tropical evergreen forest is majorly composed of actinobacteria followed by proteobacteria, firmicutes, chloroflexi, acidobacteria and bacteroidetes. Figure $3 a$ shows the top ten species relative abundance in phylum and Fig. $3 \mathrm{~b}$ shows the top ten species relative abundance in genus. The top ten phylum in the descending order of abundance includes actinobacteria, proteobacteria, firmicutes, chloroflexi, acidobacteria, verrucomicrobia, bacteroidetes, gemmatimonadetes, nitospirae and other unclassified organisms. The top ten species in the relative abundance were Bacillus, Acidothermus, Streptomyces, Solirubacter, undidentified genus, sphingomonas, pseudonocardia, geodarmatophilus, microvirga, bradyrhizobium. A majority of the genus are unclassified. Figure 4 shows the evolutionary tree of the various genus of the bacteria. Alpha diversity is applied in analyzing complexity of species diversity for a sample through 6 indices, including Observed-species, Chao1, Shannon, Simpson, ACE, Good's-coverage Table 1 shows the alpha diversity indices values for the sample.Rarefaction Curves and Rank abundance curves are widely used for indicating the biodiversity of the samples. Rarefaction Curve is created by selecting randomly certain amount of sequencing data from the samples, then counting the number of the species they represent. If the curve is steep, lots of the species remain to be discovered. If the curve becomes flatter, a credible number of samples have been taken, which means only the scarce species remain to be sampled. Rank abundance curve is used to display relative species abundance. It also can be used to visualize species richness and evenness. It overcomes the shortcomings of biodiversity indices that cannot present the role the variables played in their assessment[16] Fig. 5a shows the rarefraction curve for the sample SN1. Figure 5b shows the rank abundance curve for the sample SN1. The rarefraction curve shows that a lot of the diversity of the sample has been sampled and determined.Actinobacteria have been previously isolated from the soil samples taken from various areas including forest soil[17]. Many novel actinobacteria have been isolated from forest soil[18]. Actinobacteria are generally known to produce various bioactive compounds like antibiotics which are of both pharmaceutical and industrial relevance. Many of the bacteria isolated from 
different forest environments have been found to produce various such compounds[19,20]. Hence it is important to further study these different bacteria found in the tropical evergreen forests of India.

\section{Conclusion}

Our study shows that actinobacteria as the most abundant bacterial phylum in the soil of tropical evergreen forests of Tamil Nadu. Here in this study we understood the bacterial community composition of soils taken from a tropical evergreen forest in Tamil Nadu. This study can provide valuable insights on the soil composition, soil nutrient status, plant-microbe interactions and can also help give insights into novel compound discovery.

\section{Declarations}

\section{Ethics approval and consent to participate}

Not applicable

\section{Consent for publication}

Not applicable

\section{Availability of data and material}

Not applicable

\section{Competing interests}

There is no competing interest

\section{Funding}

There is no funding

\section{Authors' contributions}

Saptashwa Datta: Conceptualization, Methodology, Writing- Original draft preparation.

Melvin S. Samuel : Investigation, Writing- Original draft preparation.

E.Selvarajan: Supervision, Project administration

\section{References}

1. Datta, S., Rajnish, K. N., Samuel, M. S., Pugazlendhi, A., \& Selvarajan, E. (2020). Metagenomic applications in microbial diversity, bioremediation, pollution monitoring, enzyme and drug discovery. 
A review. Environmental Chemistry Letters, 18(4), 1229-1241. https://doi.org/10.1007/s10311-02001010-z

2. Oliveira, C., Gunderman, L., Coles, C. A., Lochmann, J., Parks, M., Ballard, E., ... \& Thomas, D. J. (2017). 16S rRNA gene-based metagenomic analysis of Ozark cave bacteria. Diversity, 9(3), 31. https://doi.org/10.3390/d9030031

3. Everard, M. (2018). The characteristics, representativeness, function and conservation importance of tropical dry evergreen forest on Indiaâ $€^{\text {TM }}$ s Coromandel Coast. Journal of Threatened Taxa, 10(6); 11760-11769. https://doi.org/10.11609/jott.2807.10.6.11760-11769

4. Dhinesh, K. S., Radhakrishnan, S., Balasurbramanian, A., \& Sivakumar, K. (2019). Comparative study of soil nutrient status in three forest types of Dhrampuri Forest circle, Tamil Nadu, South India. International Journal of Current Microbiology and Applied Sciences, 8(2), 16131621.https://doi.org/10.20546/ijcmas.2019.802.189

5. Magoč, T., \& Salzberg, S. L. (2011). FLASH: fast length adjustment of short reads to improve genome assemblies. Bioinformatics, 27(21), 2957-2963. https://doi.org/10.1093/bioinformatics/btr507

6. Bokulich, N. A., Subramanian, S., Faith, J. J., Gevers, D., Gordon, J. I., Knight, R., ... \& Caporaso, J. G. (2013). Quality-filtering vastly improves diversity estimates from Illumina amplicon sequencing. Nature methods, 10(1), 57-59. https://doi.org/10.1038/nmeth.2276

7. Caporaso, J. G., Kuczynski, J., Stombaugh, J., Bittinger, K., Bushman, F. D., Costello, E. K., ... \& Knight, R. (2010). QIIME allows analysis of high-throughput community sequencing data. Nature methods, 7(5), 335-336. https://doi.org/10.1038/nmeth.f.303

8. Haas, B. J., Gevers, D., Earl, A. M., Feldgarden, M., Ward, D. V., Giannoukos, G., ... \& Human Microbiome Consortium. (2011). Chimeric 16S rRNA sequence formation and detection in Sanger and 454-pyrosequenced PCR amplicons. Genome research, 21(3), 494-504. 10.1101/gr.112730.110

9. Edgar, R. C. (2013). UPARSE: highly accurate OTU sequences from microbial amplicon reads. Nature methods, 10(10), 996-998.https://doi.org/10.1038/nmeth.2604

10. Wang, Qiong, et al. Naive Bayesian classifier for rapid assignment of rRNA sequences into the new bacterial taxonomy. Applied and environmental microbiology 73.16 (2007): 5261-5267. https://doi.org/10.1093/nar/gks1219

11. Quast, C., Pruesse, E., Yilmaz, P., Gerken, J., Schweer, T., Yarza, P., ... \& Glöckner, F. O. (2012). The SILVA ribosomal RNA gene database project: improved data processing and web-based tools. Nucleic acids research, 41(D1), D590-D596

12. Edgar, R. C. (2004). MUSCLE: multiple sequence alignment with high accuracy and high throughput. Nucleic acids research, 32(5), 1792-1797 https://doi.org/10.1093/nar/gkh340

13. Asnicar, F., Weingart, G., Tickle, T. L., Huttenhower, C., \& Segata, N. (2015). Compact graphical representation of phylogenetic data and metadata with GraPhIAn. PeerJ, 3, e1029. https://doi.org/10.7717/peerj.1029/supp-1

14. Ondov, B. D., Bergman, N. H., \& Phillippy, A. M. (2011). Interactive metagenomic visualization in a Web browser. BMC bioinformatics, 12(1), 1-10. https://doi.org/10.1186/1471-2105-12-385 
15. Lundberg, D. S., Yourstone, S., Mieczkowski, P., Jones, C. D., \& Dangl, J. L. (2013). Practical innovations for high-throughput amplicon sequencing. Nature methods, 10(10), 999-1002. https://doi.org/10.1038/nmeth.2634

16. Elbendary, A. A., Hessain, A. M., El-Hariri, M. D., Seida, A. A., Moussa, I. M., Mubarak, A. S., ... \& El Jakee, J. K. (2018). Isolation of antimicrobial producing actinobacteria from soil samples. Saudi journal of biological sciences, 25(1), 44-46.https://doi.org/10.1016/j.sjbs.2017.05.003

17. Li, W. J., Xu, P., Schumann, P., Zhang, Y. Q., Pukall, R., Xu, L. H., ... \& Jiang, C. L. (2007). Georgenia ruanii sp. nov., a novel actinobacterium isolated from forest soil in Yunnan (China), and emended description of the genus Georgenia. International Journal of Systematic and Evolutionary Microbiology, 57(7), 1424-1428.https://doi.org/10.1099/ijs.0.64749-0

18. Azman, A. S., Othman, I., Fang, C. M., Chan, K. G., Goh, B. H., \& Lee, L. H. (2017). Antibacterial, anticancer and neuroprotective activities of rare Actinobacteria from mangrove forest soils. Indian journal of microbiology, 57(2), 177-187.https://doi.org/10.1007/s12088-016-0627-z

19. Das, R., Romi, W., Das, R., Sharma, H. K., \& Thakur, D. (2018). Antimicrobial potentiality of actinobacteria isolated from two microbiologically unexplored forest ecosystems of Northeast India. BMC microbiology, 18(1), 1-16.https://doi.org/10.1186/s12866-018-1215-7

\section{Figures}

a)

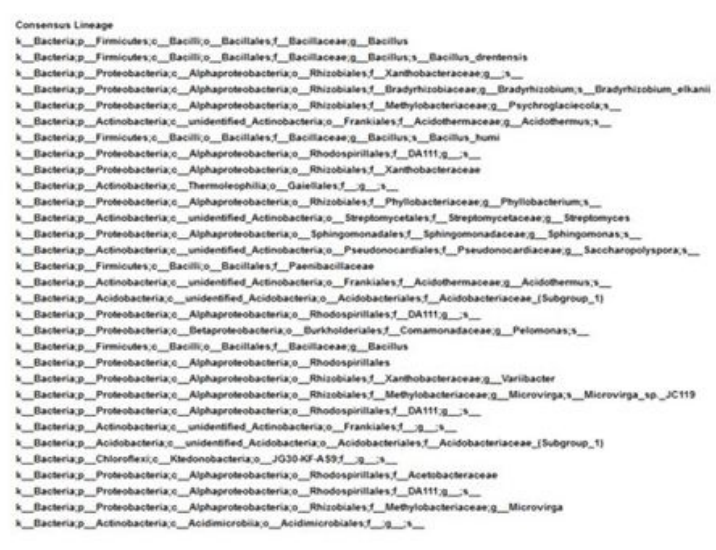

b)

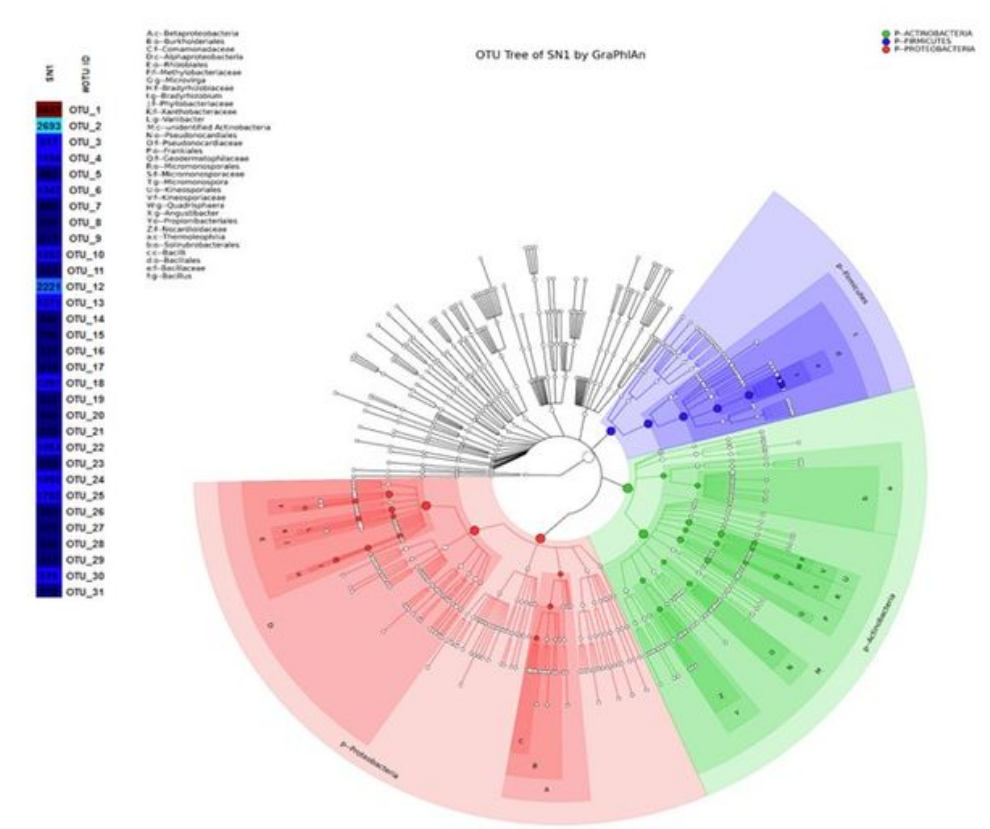

Figure 1: 1a shows the OTU table heatmap for the sample SN1. Figure 1b shows the SN1 OTU annotation tree construct using GraPhlAn

Figure 1 
1a shows the OTU table heatmap for the sample SN1. Figure 1b shows the SN1 OTU annotation tree construct using GraPhIAn

a)

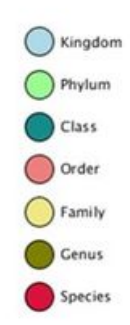

b)

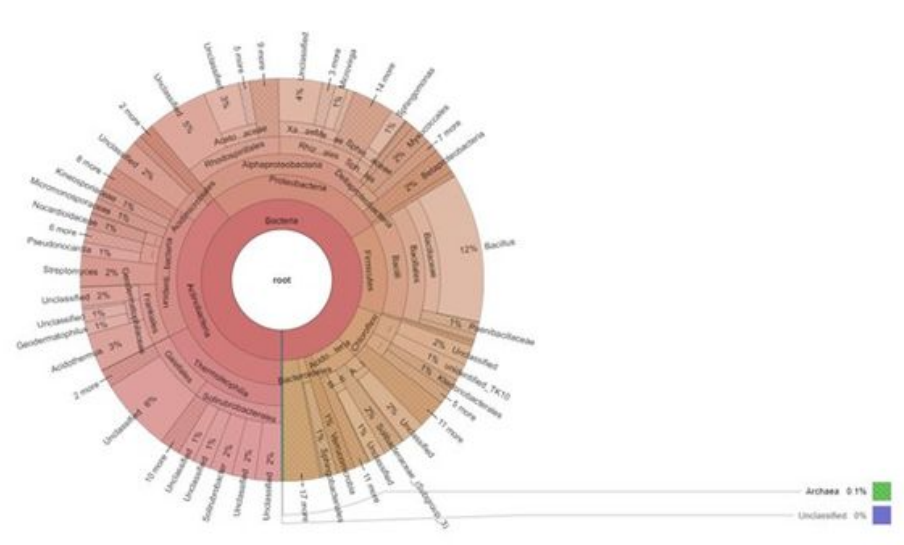

Figure 2: 2a shows the taxonomy tree of sample SN1. 2b showsthe Krona graph for sample SN1

\section{Figure 2}

2a shows the taxonomy tree of sample SN1. 2b showsthe Krona graph for sample SN1 
a)

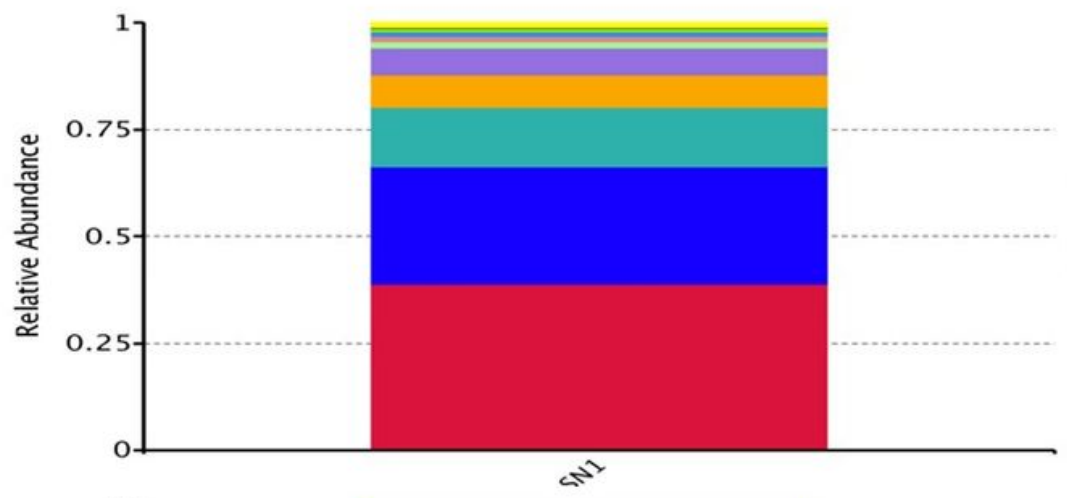

Others

N Nitrospirae

Eemmatimonadetes

Bacteroidetes

Verrucomicrobia

Chloroflexi

Firmicutes

- Proteobacteria

b)
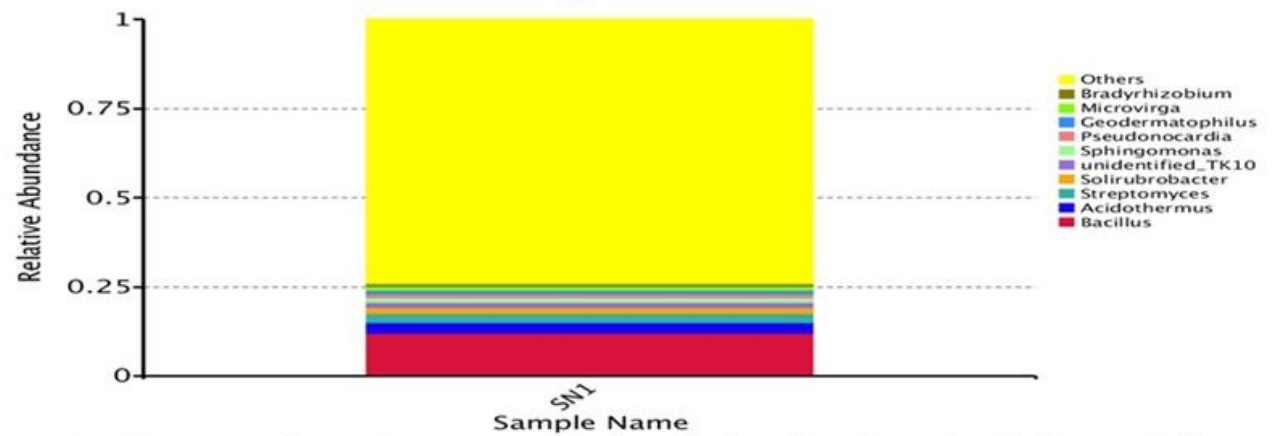

Figure 3: Figure 3a shows the top ten species relative abundance in phylum and figure $3 \mathrm{~b}$ shows the top ten species relative abundance in genus

\section{Figure 3}

Figure $3 a$ shows the top ten species relative abundance in phylum and figure $3 b$ shows the top ten species relative abundance in genus

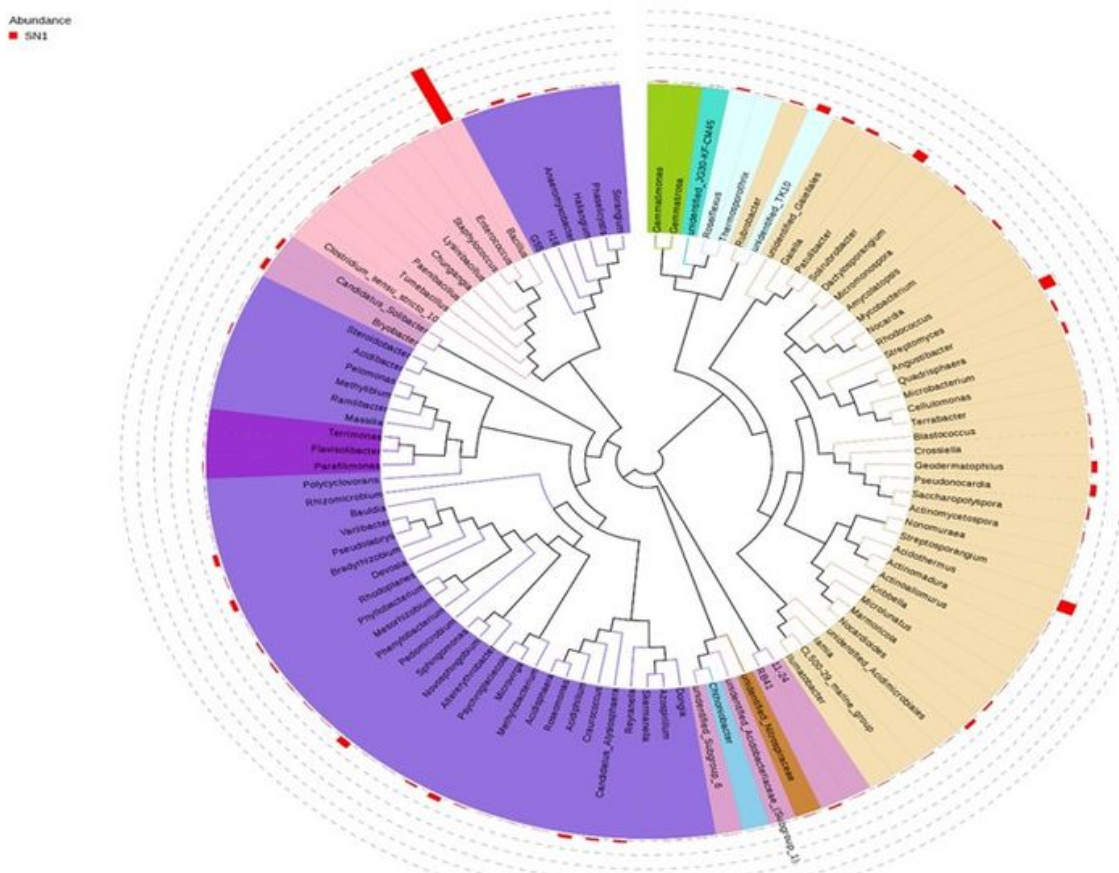

Figure 4: The evolutionary tree of the various genus of the bacteria 


\section{Figure 4}

The evolutionary tree of the various genus of the bacteria

a)

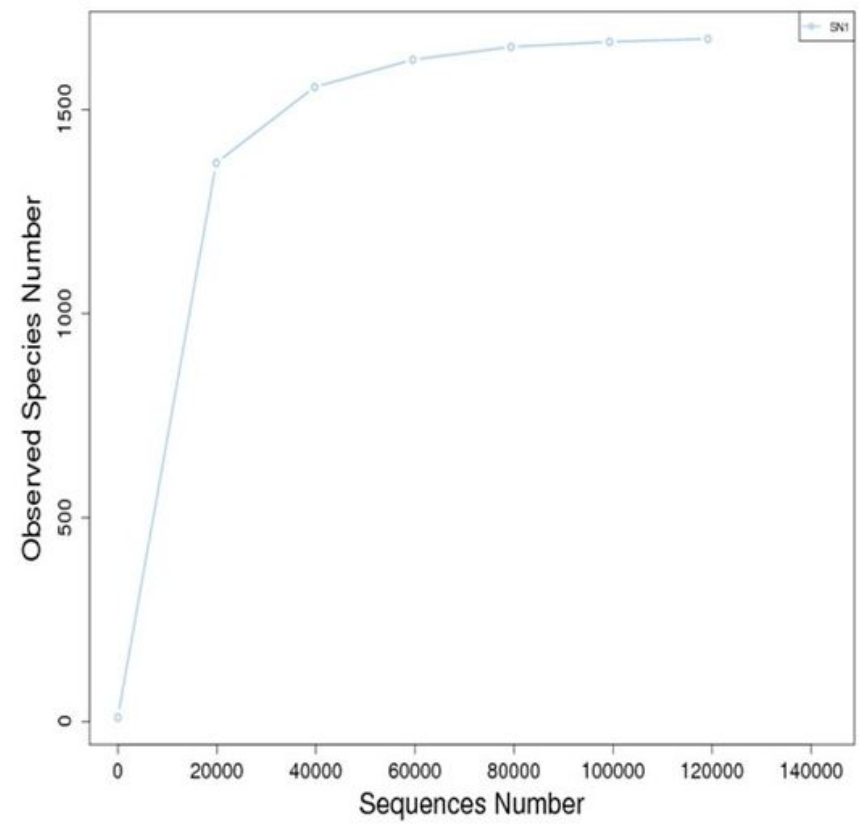

b)

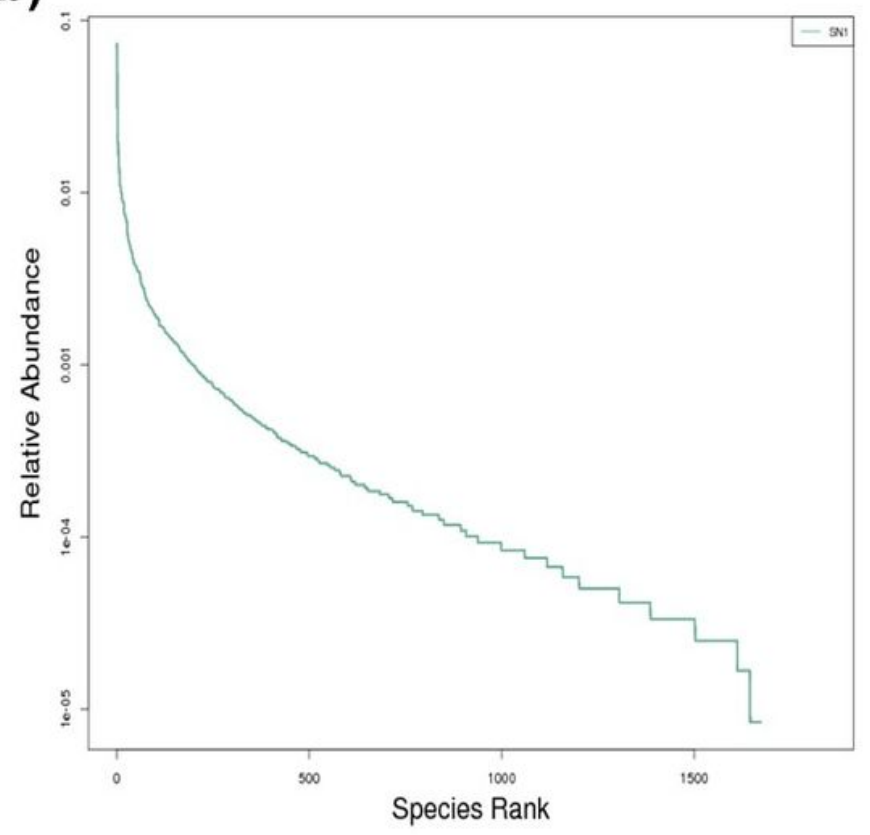

Figure 5: Figure 5a shows the rarefraction curve for the sample SN1. Figure 5b shows the rank abundance curve for the sample SN1

\section{Figure 5}

Figure 5a shows the rarefraction curve for the sample SN1. Figure 5b shows the rank abundance curve for the sample SN1 